\title{
The Association between Implementation of an Enhanced Recovery after Cesarean Pathway with Standardized Discharge Prescriptions and Opioid Use and Pain Experience after Cesarean Delivery
}

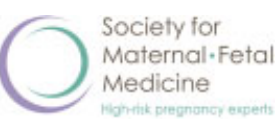

Jennifer A. McCoy, MD ${ }^{1}$ Sarah Gutman, MD, MSPH ${ }^{1} \quad$ Rebecca F. Hamm, MD ${ }^{1}$

Sindhu K. Srinivas, MD, MSCE ${ }^{1}$

${ }^{1}$ Department of Obstetrics and Gynecology, Maternal and Child Health Research Center, Perelman School of Medicine, University of Pennsylvania, Philadelphia

Address for correspondence Jennifer A. McCoy, MD, Department of Obstetrics and Gynecology, Division of Maternal Fetal Medicine, Hospital of the University of Pennsylvania, 2 Silverstein, 3400 Spruce Street, Philadelphia, PA 19104-6160

Am J Perinatol 2021;38:1341-1347.

(e-mail: jennifer.mccoy@pennmedicine.upenn.edu).

\begin{abstract}
Objective This study was aimed to evaluate opioid use after cesarean delivery (CD) and to assess implementation of an enhanced recovery after CD (ERAS-CD) pathway and its association with inpatient and postdischarge pain control and opioid use.

Study Design We conducted a baseline survey of women who underwent CD from January to March 2017 at a single, urban academic hospital. Patients were called 5 to 8 days after discharge and asked about their pain and postdischarge opioid use. An ERAS-CD pathway was implemented as a quality improvement initiative, including use of nonopioid analgesia and standardization of opioid discharge prescriptions to $\leq 25$ tablets of oxycodone of $5 \mathrm{mg}$. From November to January 2019, a postimplementation survey was conducted to assess the association between this initiative and patients' pain control and postoperative opioid use, both inpatient and postdischarge.

Results Data were obtained from 152 women preimplementation (PRE) and 137 women postimplementation (POST); complete survey data were obtained from 102 women PRE and 98 women POST. The median inpatient morphine milligram equivalents consumed per patient decreased significantly from 141 [range: $90-195$ ] PRE to 114 [range: 45-168] POST ( $p=0.002$ ). On a 0 - to 10-point scale, median patient-reported pain scores at discharge decreased significantly (PRE: 7 [range: $5-8$ ] vs. POST 5 [range: $3-7$ ], $p<0.001$ ). The median number of pills consumed after discharge also decreased significantly (PRE: 25 [range: 16-30] vs. POST 17.5 [range: $4-25$ ], $p=0.001$ ). The number of pills consumed was significantly associated with number prescribed $(p<0.001)$. The median number of leftover pills and number of refills did not

Keywords

- opioid

- cesarean

- ERAS

- pain significantly differ between groups. Median patient-reported pain scores at the week after discharge were lower in the POST group (PRE: 4 [range: $2-6$ ] vs. POST 3[range: $1-5], p=0.03$ ). Conclusion Implementing an ERAS-CD pathway was associated with a significant decrease in inpatient and postdischarge opioid consumption while improving pain control. Our data suggest that even fewer pills could be prescribed for some patients.
\end{abstract}

received

October 16, 2020

accepted after revision

June 17, 2021

published online

July 19, 2021 (c) 2021. Thieme. All rights reserved.

Thieme Medical Publishers, Inc.,

333 Seventh Avenue, 18th Floor,

New York, NY 10001, USA
DOI https://doi.org/

10.1055/s-0041-1732378.

ISSN $0735-1631$. 


\section{Key Points}

- An ERAS-CD pathway was associated with decreased opioid use.

- Outpatient opioid consumption after cesarean warrants further study.

- Physician prescribing drives patients' opioid consumption.

The United States is in the midst of an opioid use epidemic with 218,000 deaths attributable to overdoses related to prescription opioids from 1999 to $2017 .{ }^{1}$ While many factors have contributed to this epidemic, physician overprescribing bears some of the responsibility. Pain control practices surrounding cesarean delivery (CD) have attracted warranted scrutiny, as $\mathrm{CD}$ is one of the most commonly performed surgeries in the United States. Studies have shown that up to 1 in 200 to 300 women may become dependent on opioids after being exposed to them during postpartum care. ${ }^{2,3}$

Over the last several years, enhanced recovery after surgery (ERAS) pathways have been developed in multiple specialties to improve many aspects of postoperative care, including improved pain control, as well as reduction in time to postoperative mobilization, resumption of normal diet, and hospital discharge. ${ }^{4}$ These ERAS protocols have been shown to be an effective strategy to reduce overreliance on opioid pain medications for postoperative pain management. ${ }^{4-7}$ ERAS pathways implemented following gynecologic surgery have been shown to reduce inpatient opioid use and improve pain control postoperatively. ${ }^{5}$ These strategies are also increasingly being applied to patients undergoing $\mathrm{CD} .^{5,7-10}$ While reductions in inpatient opioid use have been demonstrated in obstetric, gynecologic, and general surgical patient populations, less attention has been paid to outpatient opioid use after hospital discharge. Postdischarge opioid use and pain control after CD remains understudied and warrants further attention.

Our objective was to first characterize and understand opioid prescribing practices and patients' outpatient pain medication requirements following discharge after $\mathrm{CD}$ at our institution. Our second objective was to assess the association between the implementation of an ERAS for CD (ERASCD) protocol and patients' pain control and opioid use after $C D$, both while inpatient and after hospital discharge. Our hypothesis was that the successful implementation of an ERAS-CD protocol would result in a significant reduction in inpatient and postdischarge opioid use without compromising patients' perceptions of pain control.

\section{Materials and Methods}

This study was conducted as a pre- and postimplementation quality improvement study at a large, urban, tertiary-care hospital. This study was determined to be exempt by the University of Pennsylvania institutional review board as a quality improvement initiative. A preimplementation survey of all postpartum women who had undergone CD was conducted from January through March 2017. Patients were eligible for inclusion if they delivered via CD during this time frame. Patients were excluded if they had a diagnosis of opioid use disorder in their electronic medical record or ongoing chronic opioid use documented during prenatal care.
After completion of the preimplementation survey, an ERAS-CD pathway was developed based on existing pathways for colorectal and gynecologic surgery and adopted for CD use by a working group consisting of obstetrics, anesthesiology, and nursing stakeholders. This pathway was implemented for all patients undergoing $C D$ at our institution. A major goal of the ERAS-CD pathway implementation was a reduction in the use of postpartum opioids both in the inpatient setting and upon discharge. A full description of the pain medication orders used before and after implementation of the ERAS-CD protocol is provided in -Fig. 1. Key changes including modifying the inpatient order, set from as needed (pro re nata [PRN]) to scheduled doses of nonopioid pain medications, eliminating the use of combined oxycodone-acetaminophen, and making oxycodone available only as a PRN order. Additionally, the protocol included a significant shift in the standardized discharge prescriptions for patients who underwent CD. Previously, patients were generally prescribed ibuprofen and oxycodone-acetaminophen at provider discretion. Most patients were prescribed 30 tablets of opioid medications on discharge. As part of the ERAS-CD protocol, discharge prescriptions were standardized to include ibuprofen and acetaminophen every 6 hours, and $\leq 25$ tablets of oxycodone of $5 \mathrm{mg}$ for all patients, unless there were clinical exceptions at provider discretion. The choice of $\leq 25$ tablets of 5-mg oxycodone was based on data of average opioid consumption from our preimplementation survey. Other elements of the ERAS-CD protocol were consistent with published protocol recommendations, including improved preoperative patient education, early postoperative return to PO intake and ambulation, and increased postoperative education surrounding pain control and recovery expectations. ${ }^{7-9}$

Following the successful implementation of the ERAS-CD protocol and corresponding changes in opioid prescribing

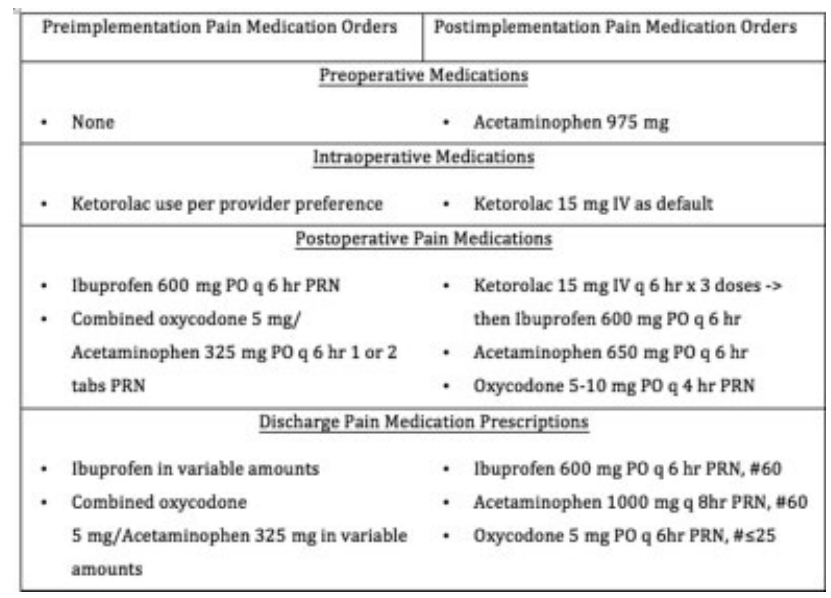

Fig. 1 Pain medication order sets pre- and postimplementation. IV, intravenous. PRN, as needed; PO, by mouth. 
practices, the same researchers conducted a postimplementation survey from November through January 2019. The same inclusion and exclusion criteria were utilized as in the preimplementation period.

In both the pre- and postimplementation surveys, all eligible patients were contacted by one of two investigators (J.A.M or S.G.) via phone call 5 to 8 days following discharge from the hospital after $\mathrm{CD}$. A standardized telephone call template, depicted in -Fig. 2, was used to assess pain intensity, ongoing use of opioids, medication storage, and plans for unused medications. If the patient reported that she had stopped using her prescribed opioids, she was asked to count the number of remaining pills. If the patient reported ongoing opioid use at the first telephone call, a second call was made 5 to 8 days later, following the same template. All opioid prescriptions were verified using the Pennsylvania Prescription Drug Monitoring Program, an online database of opioids prescriptions dispensed in the state. Demographic and clinical outcome data were abstracted from the electronic medical record.

Our coprimary outcomes were (1) inpatient morphine milligram equivalents (MMEs) consumed and (2) number of opioid pills consumed after discharge. Number of opioid pills consumed after discharge was calculated by subtracting the patient-reported number of pills remaining from the total

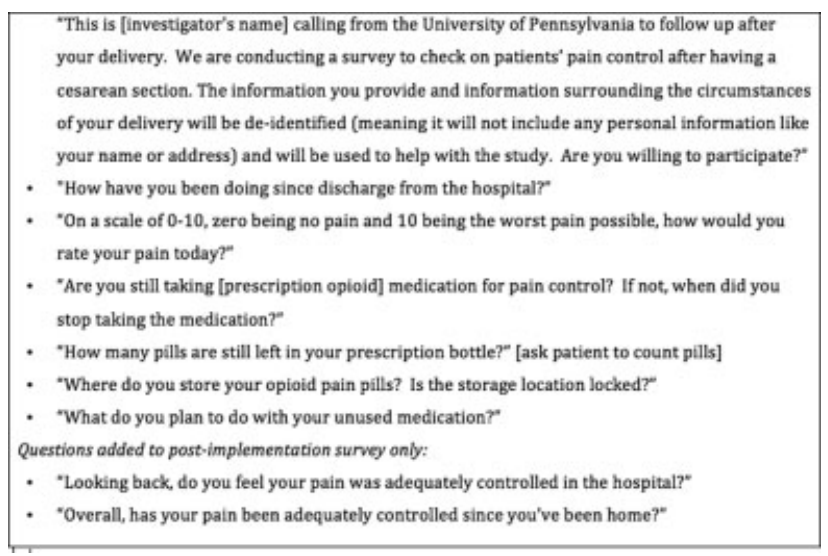

Fig. 2 Phone survey template.

number prescribed on discharge. Secondary outcomes included patient-reported pain scores at the time of discharge and at the time of first follow-up phone call. Additional secondary outcomes included number of leftover pills, number of refills needed, and patient-reported satisfaction with pain control.

For analysis, all opioid use was converted to MME according to previously published standardized conversion factors. ${ }^{11}$ Bivariate analyses were performed using Chi-square and Fisher's exact tests to compare categorical data. Student's

\begin{tabular}{|c|c|c|c|c|c|}
\hline \multirow[b]{2}{*}{ Maternal characteristic } & \multicolumn{2}{|c|}{ Preimplementation group } & \multicolumn{2}{|c|}{ Postimplementation group } & \multirow{2}{*}{$\begin{array}{l}\text { All pre- versus all } \\
\text { postimplementation, } \\
p \text {-value }\end{array}$} \\
\hline & $\begin{array}{l}\text { Reached by phone } \\
(n=102)\end{array}$ & $\begin{array}{l}\text { Not reached } \\
(n=50)\end{array}$ & $\begin{array}{l}\text { Reached by phone } \\
(n=98)\end{array}$ & $\begin{array}{l}\text { Not reached } \\
(n=39)\end{array}$ & \\
\hline $\operatorname{Age}^{a}(y)$ & $28[20-32]$ & $27[20-32]$ & $31[25-35]$ & $29[26-35]$ & $<0.001$ \\
\hline Self-identified race & & & & & 0.57 \\
\hline African-American & $66(64.7)$ & $25(58.1)$ & $64(65.3)$ & $21(58.3)$ & \\
\hline White & $24(23.5)$ & $9(20.9)$ & $25(25.5)$ & $10(27.3)$ & \\
\hline Other & $12(11.7)$ & $9(20.9)$ & $9(9.1)$ & $5(13.9)$ & \\
\hline BMI at delivery $\geq 35\left(\mathrm{~kg} / \mathrm{m}^{2}\right)$ & $35(34.3)$ & $15(34.9)$ & $38(38.8)$ & $14(38.9)$ & 0.44 \\
\hline $\begin{array}{l}\text { Hypertension (chronic or } \\
\text { pregnancy related) }\end{array}$ & $34(33.3)$ & $13(28.8)$ & 46 (46.9) & $12(33.3)$ & 0.05 \\
\hline $\begin{array}{l}\text { Diabetes (pregestational or } \\
\text { gestational) }\end{array}$ & $12(11.8)$ & $8(17.8)$ & 19 (19.4) & $6(16.7)$ & 0.19 \\
\hline $\begin{array}{l}\text { Diagnosis of } \\
\text { depression/anxiety }\end{array}$ & $17(16.7)$ & $4(8.8)$ & $17(16.3)$ & $5(13.0)$ & 0.28 \\
\hline $\begin{array}{l}\text { Any substance use disorder } \\
\text { (excluding opioids) }\end{array}$ & $6(5.9)$ & 0 & $2(2.0)$ & $3(7.6)$ & 0.20 \\
\hline Repeat cesarean delivery & $45(44.2)$ & $23(46.0)$ & $37(37.7)$ & $20(51.3)$ & 0.56 \\
\hline History of chronic pain & $4(3.9)$ & $2(3.3)$ & $1(1.0)$ & $1(3)$ & 0.28 \\
\hline Scheduled cesarean & $38(37.3)$ & 18 (41.9) & $31(31.6)$ & $13(36.1)$ & 0.27 \\
\hline Anesthesia at cesarean & & & & & 0.20 \\
\hline Spinal & $41(40.2)$ & $13(30.3)$ & $43(43.9)$ & $14(38.9)$ & \\
\hline Epidural & $51(50.0)$ & $26(60.4)$ & $49(50.0)$ & $22(61.1)$ & \\
\hline General & $10(9.8)$ & $4(9.3)$ & $6(6.1)$ & 0 & \\
\hline
\end{tabular}

Abbreviation: BMI, body mass index.

Note: All data are presented as $n(\%)$ unless otherwise indicated.

${ }^{a}$ Median [interquartile range]. 
Table 2 Opioid outcomes by group

\begin{tabular}{|c|c|c|c|c|c|}
\hline \multirow[b]{2}{*}{ Outcome } & \multicolumn{2}{|c|}{ Preimplementation group } & \multicolumn{2}{|c|}{ Postimplementation group } & \multirow{2}{*}{$\begin{array}{l}\text { All pre- versus all } \\
\text { postimplementation, } p \text {-value }\end{array}$} \\
\hline & $\begin{array}{l}\text { Reached by } \\
\text { phone }(n=102)\end{array}$ & $\begin{array}{l}\text { Not reached } \\
(n=50)\end{array}$ & $\begin{array}{l}\text { Reached by } \\
\text { phone }(n=98)\end{array}$ & $\begin{array}{l}\text { Not reached } \\
(n=39)\end{array}$ & \\
\hline \multicolumn{6}{|l|}{ Inpatient outcomes } \\
\hline Total inpatient MME & $142.5[90-195]$ & 135 [105-187.5] & $112.5[45-150]$ & $120[41-180]$ & 0.02 \\
\hline $\begin{array}{l}\text { Patients who used no opioids } \\
\text { inpatient }^{\mathrm{a}}\end{array}$ & $4(3.9)$ & 0 & $9(9.18)$ & $4(10.3)$ & 0.01 \\
\hline Pain score at hospital discharge & $7[5-8]$ & $6[5-8]$ & $5[3-7]$ & $5.5[3.5-7]$ & $<0.001$ \\
\hline Opioid prescribed ${ }^{\mathrm{a}}$ & & & & & $<0.001$ \\
\hline Oxycodone & 0 & $0(0.0)$ & $89(90.8)$ & $32(88.9)$ & \\
\hline Oxycodone/acetaminophen & $92(90.1)$ & $48(96.0)$ & 0 & 0 & \\
\hline Hydromorphone & $7(6.7)$ & $2(4.0)$ & $9(9.2)$ & $4(11.1)$ & \\
\hline Other & $1(1.0)$ & 0 & 0 & 0 & \\
\hline $\begin{array}{l}\text { Number of pills in discharge } \\
\text { prescription }\end{array}$ & $30[30-30]$ & $30[30-30]$ & $25[25-25]$ & $25[25-25]$ & $<0.001$ \\
\hline \multicolumn{6}{|l|}{ Postdischarge outcomes } \\
\hline Number of pills consumed & $25[16-30]$ & - & $17.5[4-25]$ & - & $<0.001$ \\
\hline Total MME after discharge & $38.8[27-45]$ & - & $28.5[6-37.5]$ & - & $<0.001$ \\
\hline Number of pills leftover & $5[0-15]$ & - & $3[0-18]$ & - & 0.83 \\
\hline Took all prescribed pills ${ }^{a}$ & $44(43.1)$ & - & $38(38.8)$ & - & 0.53 \\
\hline Took no prescribed pills ${ }^{a}$ & $7(6.9)$ & - & $18(18.4)$ & - & 0.01 \\
\hline Kept pills in locked location ${ }^{a}$ & $8(7.8)$ & - & $8(8.1)$ & - & 0.80 \\
\hline Received opioid refilla & $6(5.9)$ & - & $1(1.0)$ & - & 0.12 \\
\hline Pain score at home first call & $4[2-6]$ & - & $3[1-5]$ & - & 0.03 \\
\hline
\end{tabular}

Abbreviation: MME, morphine milligram equivalents.

Note: All data are presented as median [interquartile range] unless otherwise indicated.

${ }^{a} n(\%)$.

$t$-test and Mann-Whitney $U$-test were used to compare continuous parametric and nonparametric data, where appropriate. Proportional odds regression was used to evaluate factors associated with postdischarge opioid use. To create the final model, backward selection was used, and covariates with a $p$-value of $<0.05$ were considered statistically significant and kept in the model. All statistical analyses were performed using STATA version 15.1 (College Station, TX).

Our sample size calculation was based on our coprimary outcome of number of opioid pills consumed after discharge. Using $80 \%$ power and an $\alpha$ of 0.05 , we determined that we would need 100 patients per group to detect a clinically meaningful five-pill decrease in mean number of pills consumed postdischarge between the pre- and postimplementation groups.

\section{Results}

In the preimplementation period, 152 eligible patients were called and 102 were successfully reached and included for analysis, a survey response rate was $67 \%$ (PRE). In the postimplementation period, 137 eligible women were called and 98 were successfully reached and included for analysis, a survey response rate of $71 \%$ (POST). The demographic and clinical data for both PRE and POST survey participants, subdivided by those who were reached for the phone survey and those who were not, are shown in - Table 1. Overall, the PRE and POST groups differed only in median age, as shown in the $p$-values in - Table 1 . When comparing within the PRE and POST groups, there were no significant demographic differences between those eligible patients who were reached and those who were not. When comparing those patients who were reached for the phone survey between PRE and POST, the groups were similar: the majority of patients self-identified as African American (64.7\% PRE vs. 65.3\% POST, $p=0.75$ ), approximately one-third had a body mass index $>35 \mathrm{~kg} / \mathrm{m}^{2}$ ( 34.3 vs. $38.5 \%, p=0.51$ ), and the majority underwent a primary CD (55.8 vs. $62.3 \%, p=0.36)$ and had an unscheduled CD (62.7 vs. $68.4 \%, p=0.40)$. The vast majority of patients received regional anesthesia for their CD in both groups (90.2\% PRE and 93.9\% POST, $p=0.60$ ). The groups were significantly different in median age (28 years PRE vs. 31 years POST, $p<0.001$ ).

Outcomes related to opioid use are shown in - Table 2. In the inpatient setting, the median MME of opioids consumed per patient decreased significantly between the entire PRE and POST groups (141 [range: 90-195] vs. 114 [range: 45-168], $p=0.002$ ). The percentage of patients who used no opioids as inpatients also increased significantly from PRE to POST $(2.6 \%$ PRE vs. 9.5\% POST, $p=0.01)$. Patient-reported pain scores as reported to their nurse on their final assessment at the time of hospital discharge decreased from a median of 7 [range: 5-8] 


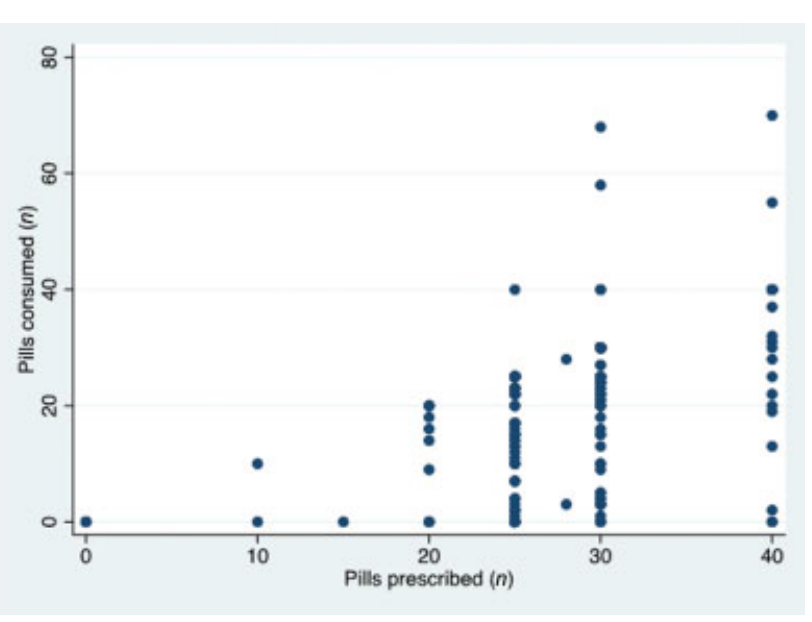

Fig. 3 Plot of the relationship between the number of opioid pills prescribed after hospital discharge from cesarean delivery and the number of pills consumed.

PRE to 5 [range: $3-7]$ POST on a 0 - to 10 -point scale $(p<0.001)$. Opioid discharge prescriptions changed significantly, in accordance with the successful implementation of the ERAS-CD protocol. In the PRE group, $91 \%$ of discharge prescriptions were combined oxycodone/acetaminophen. In the POST group, 88\% of prescriptions were for oxycodone alone. In the PRE group, $96 \%$ of patients were prescribed $\geq 30$ pills. In contrast, in the POST group, $97 \%$ of patients were prescribed $\leq 25$ pills.

When evaluating the data obtained from those patients who completed the postdischarge phone survey, the median number of opioid pills consumed after hospital discharge was significantly lower in the POST group (25 [range: 16-30] PRE vs. 17.5 [range: 425 ] POST, $p<0.001)$. Patients in the POST group consumed a median of 7.5 fewer opioid pills after discharge compared with patients in the PRE group. The number of patients who consumed all pills prescribed was similar between groups, at 43.1\% PRE and $38.8 \%$ POST ( $p=0.53$ ). The median number of leftover pills was also similar between groups (5 [range: 0-15] PRE vs. 3 [range: 018 ] POST, $p=0.83$ ). The number of patients who took no opioids at home increased significantly in the POST group (6.9\% PRE-$18.4 \%$ POST $p=0.01$ ). The number of patients who received opioid prescription refills decreased from PRE to POST, but this did not reach statistical significance (5.9\% PRE vs. $1.0 \%$ POST, $p=0.12$ ).

Patient-reported pain scores as reported on phone survey 5 to 8 days after discharge decreased significantly among patients from the PRE period compared with the POST, as measured on a 0 - to 10 -point scale ( 4 [range: $2-6$ ] vs. 3 [1-5], $p=0.03$ ). When patients in the POST group were asked if they felt that their pain had been adequately controlled in the hospital, 95\% answered yes. When asked if their pain had been adequately controlled since hospital discharge, $88 \%$ of patients in the POST group answered yes. These questions were not asked of the PRE group, so data are not available for comparison.

-Fig. 3 depicts the relationship between the number of opioid pills prescribed after hospital discharge from CD and the number of pills consumed. In a multivariable proportional odds regression model controlling for parity, the number of opioid pills consumed after hospital discharge was significantly associated with the number of pills pre- scribed in both the PRE and POST groups. For every five additional pills prescribed, one additional pill was consumed (adjusted odds ratio $[\mathrm{aOR}]=1.20, p<0.001$ ).

Among both groups, only $9 \%$ of patients reported that they kept their pills in a locked location in their home. When women were asked what they planned to do with any remaining pills, $28 \%$ replied that they would keep them for future use and only $25 \%$ had a plan to dispose of them.

\section{Discussion}

In this study, we sought to describe postpartum opioid use and pain control among patients undergoing $\mathrm{CD}$ and to evaluate the association between implementation of an ERAS-CD protocol and these outcomes. We demonstrated that successful implementation of an ERAS-CD protocol was associated with a decrease in quantity of opioids used both during inpatient hospitalization and after hospital discharge. Furthermore, we found that this decrease in opioid usage was accompanied by a significant improvement in patientreported pain scores both at the time of hospital discharge and after 1 week at home, and that patients reported that their pain had been adequately controlled. Finally, we demonstrated that the majority of patients had extra opioid pills leftover, but did not have a safe plan to dispose of them. These findings demonstrate that we can decrease patients' opioid use after $C D$ while simultaneously improving their perceived pain control, but that more work is needed to reduce the risks of excess opioids remaining in homes.

This study is one of the few to examine patients' opioid use and pain control in the weeks after hospital discharge. ${ }^{2,6,12}$ Our findings are unique in demonstrating an association between ERAS-CD implementation and decreased opioid use and improved pain control at 1 week following hospital discharge. and Osmundson et al and Bateman et al had previously found that nonstandardized postdischarge opioid prescriptions after CD often exceed individual's pain requirements. ${ }^{6,12}$ Our data showed that after the successful implementation of reduced opioid prescriptions, patients consumed a median of 7.5 fewer opioid pills after discharge, yet their pain control improved. Notably, despite the decreased number of pills prescribed and consumed overall, the number of pills leftover at home and the number of refills requested did not change. We also demonstrated that the number of pills prescribed was significantly associated with the number of pills consumed across both study groups. As a whole, these data suggest that patients' opioid consumption is meaningfully influenced by the number of pills prescribed. Consistent with prior evidence from Bateman et al, our data demonstrate that physician prescribing shapes patients' expectations regarding their pain control and medication needs. ${ }^{12}$ As ERAS protocols continue to gain in popularity and further reductions in opioid prescribing are explored, our work contributes significantly to the, thus, far understudied area of how these protocols are impacting pain control and opioid use after discharge.

Furthermore, our findings of decreased inpatient opioid usage following implementation of an ERAS protocol are consistent with numerous other studies. ${ }^{7,13-16}$ Additionally, 
we noted a significant increase in the number of patients who did not use any opioids during their inpatient postpartum stay, though our study is underpowered to specifically assess pain control outcomes in this subgroup.

Overall, our work supports that idea that we can effectively decrease our patients' postpartum opioid use by optimizing nonopioid analgesics and prescribing reduced quantities of opioids. This evidence should empower clinicians to reduce the number of opioid tablets prescribed without concern that they are inadequately managing their patients' pain. Furthermore, our findings demonstrate that providers can significantly decrease the number of opioid pills they are prescribing without leading to an increase in prescription refills. This should provide reassurance to providers who are motivated to decrease patients' postoperative opioid consumption that they can do so without compromising patients' pain control.

Our findings have numerous implications for further research. The fact that most patients had leftover pills and fewer than half of patients consumed all their prescribed pills suggest that the number of tablets prescribed could be decreased even afterward. Further study is needed to assess whether adequate pain control could be achieved with even fewer pills prescribed, or with individualized prescribing fit to each patient's personal needs. Some institutions have decreased prescriptions through shared decision-making or standardized prescriptions of fewer than 20 tablets, and since the implementation of this study, our institution has further reduced our standardized prescription to 20 tablets of 5-mg oxycodone., $6,17,18$ We anticipate that additional research may demonstrate that very low amounts of opioid medication can effectively control both inpatient and postdischarge postoperative cesarean pain when part of ERAS protocols utilizing multimodal analgesia.

Additionally, our data show that only a minority of patients were properly storing and disposing of their leftover opioid pills. This offers an opportunity for improved patient education, surrounding safe management of opioids in the home and safe disposal options. During the course of our study implementation, there was not a standardized process for instructing patients on opioid storage and disposal. The lack of consistent patient education is a common finding within studies addressing postpartum opioid prescriptions. ${ }^{2,6,19}$ An evaluation of discharge counseling demonstrated increased rates of proper opioid disposal among patients who received standardized counseling prior to discharge after cesarean section. ${ }^{19}$ Additional research in this area could improve storage and disposal practices across hospital settings.

\section{Strengths and Limitations}

Our study has several strengths. It was performed prospectively, and we contacted patients to assess their perceptions of pain control at home after delivery. The assessment of the impact of a postcesarean ERAS protocol on both inpatient and postdischarge outcomes is a previously understudied area. We used standard interviews to evaluate number of leftover pills, instead of relying on indirect metrics, such as prescription refills. Additionally, our use of the Pennsylvania Drug Monitoring Program, which compiles all opioid prescriptions filled in the state of Pennsylvania, allows us to ensure that we captured any prescriptions that a patient might have obtained from other in-state providers. Furthermore, the majority of our patients self-identify as African American, an understudied population that is particularly vulnerable to disparities in postpartum pain management. ${ }^{20,21}$

Our study also has several limitations. It was conducted at one hospital center which limits the generalizability of our findings. However, implementation of an ERAS protocol and reductions in the number of pills prescribed at discharge are translatable to all hospital settings. Overall, we successfully reached $69 \%$ of the patients eligible for our study. It is possible that those patients we did not reach differ from the study participants in their postdischarge opioid consumption. However, the demographic and inpatient data between those reached and not reached were similar, making this unlikely to affect our findings. Additionally, the use of a phone interview to determine pain scores at home and number of pills consumed could be vulnerable to reporting or social desirability bias if patients felt compelled to provide answers that they thought would be acceptable to the researcher. However, since the same survey methods were used for both the PRE and POST data collection, this possible bias should not change our results. Finally, it is possible that other secular trends or unmeasured changes between our PRE and POST periods could have affected our findings. For instance, there has been increasing attention in the lay press to the opioid crisis and risks of opioid addiction, and this could have impacted patients' opioid use.

\section{Conclusion}

Our findings demonstrate that implementing an ERAS-CD protocol and prescribing a smaller number of opioids at discharge were associated with a decrease in both inpatient opioid use, as well as number of opioid pills consumed after hospital discharge. Furthermore, our data demonstrate that these changes can be accomplished while actually improving patients' postoperative pain control. Further work is needed to validate these findings in other patient populations to further study the implementation of ERAS-CD protocols in other clinical settings and to determine whether even further decreases in opioid prescribing can improve outcomes.

\section{Note}

This work was presented at the Society for Maternal-Fetal Medicine's 40th Annual Pregnancy Meeting, Grapevine, Texas, February 3-8, 2020.

Conflict of Interest

None declared.

\section{References}

1 Centers for Disease Control. Prescription opioid data. Accessed July 13, 2020 at: https://www.cdc.gov/drugoverdose/data/ prescribing.html

2 Bateman BT, Franklin JM, Bykov K, et al. Persistent opioid use following cesarean delivery: patterns and predictors among 
opioid-naïve women. Am J Obstet Gynecol 2016;215(03):353. e1-353.e18

3 Peahl AF, Dalton VK, Montgomery JR, Lai YL, Hu HM, Waljee JF. Rates of new persistent opioid use after vaginal or cesarean birth among US women. JAMA Netw Open 2019;2(07):e197863

4 Steenhagen E. Enhanced recovery after surgery: it's time to change practice!. Nutr Clin Pract 2016;31(01):18-29

5 Nelson G, Bakkum-Gamez J, Kalogera E, et al. Guidelines for perioperative care in gynecologic/oncology: Enhanced Recovery after Surgery (ERAS) Society recommendations-2019 update. Int J Gynecol Cancer 2019;29(04):651-668

6 Osmundson SS, Schornack LA, Grasch JL, Zuckerwise LC, Young JL, Richardson MG. Postdischarge opioid use after cesarean delivery. Obstet Gynecol 2017;130(01):36-41

7 Hedderson M, Lee D, Hunt E, et al. Enhanced recovery after surgery to change process measures and reduce opioid use after cesarean delivery: a quality improvement initiative. Obstet Gynecol 2019;134(03):511-519

8 Wilson RD, Caughey AB, Wood SL, et al. Guidelines for antenatal and preoperative care in cesarean delivery: Enhanced Recovery After Surgery Society recommendations (part 1). Am J Obstet Gynecol 2018;219(06):523.e1-523.e15

9 Macones GA, Caughey AB, Wood SL, et al. Guidelines for postoperative care in cesarean delivery: Enhanced Recovery After Surgery (ERAS) Society recommendations (part 3). Am J Obstet Gynecol 2019;221(03):247.e1-247.e9

10 Caughey AB, Wood SL, Macones GA, et al. Guidelines for intraoperative care in cesarean delivery: Enhanced Recovery After Surgery Society recommendations (part 2). Am J Obstet Gynecol 2018;219(06):533-544

11 Centers for Disease Control. Calculating total daily dose of opioids for safer dosage. Accessed July 13, 2020 at: https://www.cdc.gov/ drugoverdose/pdf/calculating_total_daily_dose-a.pdf
12 Bateman BT, Cole NM, Maeda A, et al. Patterns of opioid prescription and use after cesarean delivery. Obstet Gynecol 2017;130 (01):29-35

13 Smith AM, Young P, Blosser CC, Poole AT. Multimodal stepwise approach to reducing in-hospital opioid use after cesarean delivery: a quality improvement initiative. Obstet Gynecol 2019;133 (04):700-706

14 Holland E, Bateman BT, Cole N, et al. Evaluation of a quality improvement intervention that eliminated routine use of opioids after cesarean delivery. Obstet Gynecol 2019;133(01):91-97

15 Mullman L, Hilden P, Goral J, et al. Improved outcomes with an enhanced recovery approach to cesarean delivery. Obstet Gynecol 2020;136(04):685-691

16 Meyer LA, Lasala J, Iniesta MD, et al. Effect of an enhanced recovery after surgery program on opioid use and patientreported outcomes. Obstet Gynecol 2018;132(02):281-290

17 Prabhu M, McQuaid-Hanson E, Hopp S, et al. A shared decisionmaking intervention to guide opioid prescribing after cesarean delivery. Obstet Gynecol 2017;130(01):42-46

18 Lakhi N, Tricorico G, Kanninen T, Suddle R, Ponterio J, Moretti M. Post-cesarean delivery outpatient opioid consumption and perception of pain control following implementation of a restrictive opioid prescription protocol. Am J Obstet Gynecol MFM 2019;1 (04):100049

19 Lam L, Richardson MG, Zhao Z, Thampy M, Ha L, Osmundson SS. Enhanced discharge counseling to reduce outpatient opioid use after cesarean delivery: a randomized clinical trial. Am J Obstet Gynecol MFM 2021;3(01):100286

20 Badreldin N, Grobman WA, Yee LM. Racial disparities in postpartum pain management. Obstet Gynecol 2019;134(06):1147-1153

21 Johnson JD, Asiodu IV, McKenzie CP, et al. Racial and ethnic inequities in postpartum pain evaluation and management. Obstet Gynecol 2019;134(06):1155-1162 